\title{
Presencia y Distribución de Tabiques Intrasinusales en el Piso del Seno Maxilar
}

\author{
Prescence and Distribution of Intrasinusal Septa in the Maxillary Sinus Floor
}

*,**Arnoldo Hernández Caldera; **Anette Vistoso Monreal; "**Rodrigo Hernández Quezada; **Javier Rojo Pereira

HERNANDEZ, C. A.; VISTOSO, M. A; HERNANDEZ, Q. R. \& ROJO, P. J. Presencia y distribución de tabiques intrasinusales en el piso del seno maxilar. Int. J. Morphol., 29(4):1168-1173, 2011.

RESUMEN: La pérdida de dientes superiores causa reabsorción del proceso alveolar y la neumatización del seno maxilar. La implantología oral ha permitido solucionar la perdida de dientes, sin embargo, cuando existe neumatización del seno maxilar, la disponibilidad ósea se ve disminuida, dificultando el procedimiento implantologico. Para remediar esta situación se efectúa la técnica quirúrgica de levantamiento del piso del seno maxilar, la que puede tener complicaciones por la morfología interna del seno, específicamente por la presencia de septos intrasinusales. El objetivo de este estudio es verificar la presencia y distribución de los septos intrasinusales, debido a su importancia en técnicas quirúrgicas realizadas en implantología oral. Se realizó un estudio descriptivo, basado en el análisis visual de huesos maxilares aislados. De 65 huesos se seleccionaron 51(42 dentados y 9 edéntulos) que cumplían con determinados criterios de inclusión. La segunda parte del estudio consistió en dividir topográficamente el piso del seno en tres regiones: anterior a la cresta cigomato alveolar, en relación a ella, y posterior a la cresta. La observación de los septos fue realizado por un único examinador, asistido por un dispositivo USB, con 4 leds de alta luminiscencia. De los 51 maxilares analizados se obtuvo: 74,5\%, presentaron al menos un septo intrasinusal, 25,4\%, no presentaron septos visibles. Los maxilares que presentaron un único tabique correspondieron al $33,3 \%$ de la muestra, el 19,6 \% de la muestra presento sólo dos tabiques, el 15,7\% presentó tres tabiques, mientras que los maxilares que presentaron más de tres tabiques intrasinusales correspondieron sólo al 5,9\%. Del total de tabiques encontrados (75 tabiques) el $42 \%$ se observó en la región anterior, $21 \%$ en la región de la cresta cigomato alveolar y el 37 \% en la región posterior del seno maxilar. De los 42 maxilares en condición dentada el 88,1\% presento tabiques intrasinusales, mientras que del total de los maxilares edéntulos (9) solo el $11 \%$ presento tabiques intrasinusales. Este estudio establece que un porcentaje importante de los huesos maxilares poseen septos intrasinusales que modifican la morfología del piso sinusal con una frecuencia y ubicación variable. Esta situación deberá ser considerada y evaluada mediante imagenología en la realización de técnicas quirúrgicas de elevación del piso del seno maxilar.

PALABRAS CLAVE: Seno maxilar; Tabiques intrasinusales; Elevación del piso del seno maxilar.

\section{INTRODUCCIÓN}

La pérdida de piezas dentarias determina cambios morfológicos y funcionales importantes a nivel del sistema cráneo cervicomandibular. Estos cambios se pueden ver reflejados tanto a nivel oral como a nivel extraoral, siendo estos últimos dependientes de la ubicación de la pieza perdida. Los principales cambios morfológicos a nivel oral se establecen por la pérdida progresiva del proceso alveolar tanto en altura como en grosor (Gosau et al., 2009; Lee et al., 2010; Raja, 2009). El patrón de reabsorción del proceso alveolar varía si la pérdida de la pieza dentaria es superior o inferior, ya que el patrón reabsortivo maxilar se describe como centrípeto a diferencia del patrón reabsortivo mandibular que se describe como centrifugo.
El arco dentario en el maxilar presenta relaciones anatómicas distintas dependiendo de la pieza dentaria, reconociéndose 4 grupos dentarios: a) en relación al piso de la cavidad nasal, siendo estos fundamentalmente el grupo incisivo. b) en relación al pilar canino, vale decir por lateral a la pared lateral de la cavidad nasal y por medial a la pared medial y anterior del seno maxilar, se describe en esta región al diente canino; c) en relación al piso del seno maxilar, a este grupo pertenecen los premolares y los primeros y segundos molares; d) en relación a la tuberosidad del maxilar, encontrándose en este grupo fundamentalmente al tercer molar. Estas relaciones anatómicas pueden variar dependiendo del grado de neumatización del seno maxilar, si-

\footnotetext{
*Programa de Anatomía y Biología del Desarrollo, Instituto de Ciencias Biomédicas, Facultad de Medicina, Universidad de Chile, Santiago, Chile.

**Facultad de Odontología, Universidad de Chile, Santiago, Chile.
} 
tuación que se ve exacerbada al perder un diente, describiéndose neumatizaciones tanto hacia la pared anterolateral del seno como también hacia la tuberosidad del maxilar. De este modo la altura del proceso alveolar se ve alterada tanto por la reabsorción alveolar como también por la neumatización del seno maxilar (Gosau et al.; Kim et al., 2006; Lee et al.; Maestre-Ferrín et al., 2010; Raja).

El seno maxilar corresponde a una cavidad neumática ubicada en el tercio medio del rostro, específicamente al interior de hueso maxilar y cuyo tamaño varia entre los distintos individuos y a las distintas edades (Farah \& Nafis, 2006; Lawson et al., 2008; Park et al., 2010). En su piso es posible encontrar una serie de accidentes óseos descritos como tabiques intrasinusales, los cuales corresponden a elevaciones óseas lineales que transcurren desde una pared del seno maxilar hacia la otra (Gosau et al.; Kim et al.; Krennmair et al., 1999; Maestre-Ferrín et al.; McGowan, 1993; Underwood, 1910; Velásquez-Plata et al., 2002). La presencia de tabiques intrasinusales en las distintas porciones del piso del seno maxilar determina un factor de riesgo en las variadas técnicas de elevación del piso del seno maxilar, ya que estos y debido a su morfología lineal y muchas veces cortante, pueden llegar a desgarrar a la mucosa que tapiza el interior del seno maxilar con el consecuente sangrado y complicación operatoria del momento (Chanavaz, 1990; Ella et al., 2008; Gosau et al.; Greenstein et al., 2008; Shibli et al., 2007; Ulm et al., 1995; Van den Bergh et al., 2000).

El objetivo de este trabajo es comprobar la presencia y la distribución de los tabiques intrasinusales en el piso del seno maxilar en piezas óseas cadavéricas. El estudio morfológico interno del seno maxilar debiera ser considerado en los protocolos clínicos y radiográficos, con el fin de disminuir las posibles complicaciones en las técnicas de elevación del piso del seno maxilar.

\section{MATERIAL Y MÉTODO}

Se realizó un estudio descriptivo en el laboratorio de Anatomía Humana de Cabeza y Cuello del Programa de Anatomía y Biología del Desarrollo de la Facultad de Medicina de la Universidad de Chile.

La unidad de muestra quedo determinada por piezas óseas de maxilares aislados. De un total de 65 maxilares se seleccionaron 51 maxilares que cumplían con los siguientes criterios de inclusión:

- Integridad de la pared lateral y piso del seno maxilar.
- Libres de articulación con otros huesos vecinos (palatino, esfenoides, etmoides).

De los 51 huesos seleccionados, 42 de ellos conservaban a los menos 2 piezas dentarias, mientras que 9 de los maxilares fueron clasificados como edentulos totales.

Posterior a esto se procedió a dividir topográficamente el piso del seno maxilar en 3 regiones de acuerdo a la proyección de la cresta cigomato alveolar hacia el interior del seno y específicamente en su proyección en el piso desde la pared lateral hacia la pared medial, siendo esta proyección perpendicular al eje anteroposterior mayor del mismo piso. De este modo el piso se dividió en las siguientes regiones:

- Anterior a la proyección de la cresta cigomato alveolar.

- En relación a la cresta cigomato alveolar.

- Posterior a la proyección de la cresta cigomato alveolar.

Una vez clasificada topográficamente la región del piso del seno maxilar se procedió a realizar el examen visual del mismo, apoyándose en un dispositivo USB con 4 leds de alta luminiscencia. El examen visual fue realizado por un único examinador. Los tabiques observados en cada uno de los maxilares fueron clasificados de acuerdo a su ubicación topográfica y al mismo tiempo se registró el número de tabiques presentes en cada maxilar.

\section{RESULTADOS}

Del total de maxilares observados y analizados (51 casos) el 74,5\% de ellos presentó a lo menos un tabique a nivel del piso del seno maxilar, siendo estos de variadas morfologías y grosores pero conservando un patrón común de dirección, el que se proyecta desde la pared medial a la lateral en sentido perpendicular al eje mayor antero posterior del piso del seno (Fig. 1).

En cuanto a la frecuencia de los tabiques en el piso de los maxilares analizados se pudo establecer que el porcentaje de maxilares que presentaban tan solo 1 tabique con respecto al total de maxilares estudiados corresponde al 33\%; Aquellos maxilares que presentaron 2 tabiques en su piso correspondieron al 19,6\%; la presencia de 3 tabiques en el piso del seno representa un 15,7\%; en tanto que aquellos maxilares que presentaron mas allá de 3 tabiques intrasinusales representaron el 5,9\% del total de la muestra seleccionada (Fig. 2).

La distribución de los 75 tabiques encontrados en el 


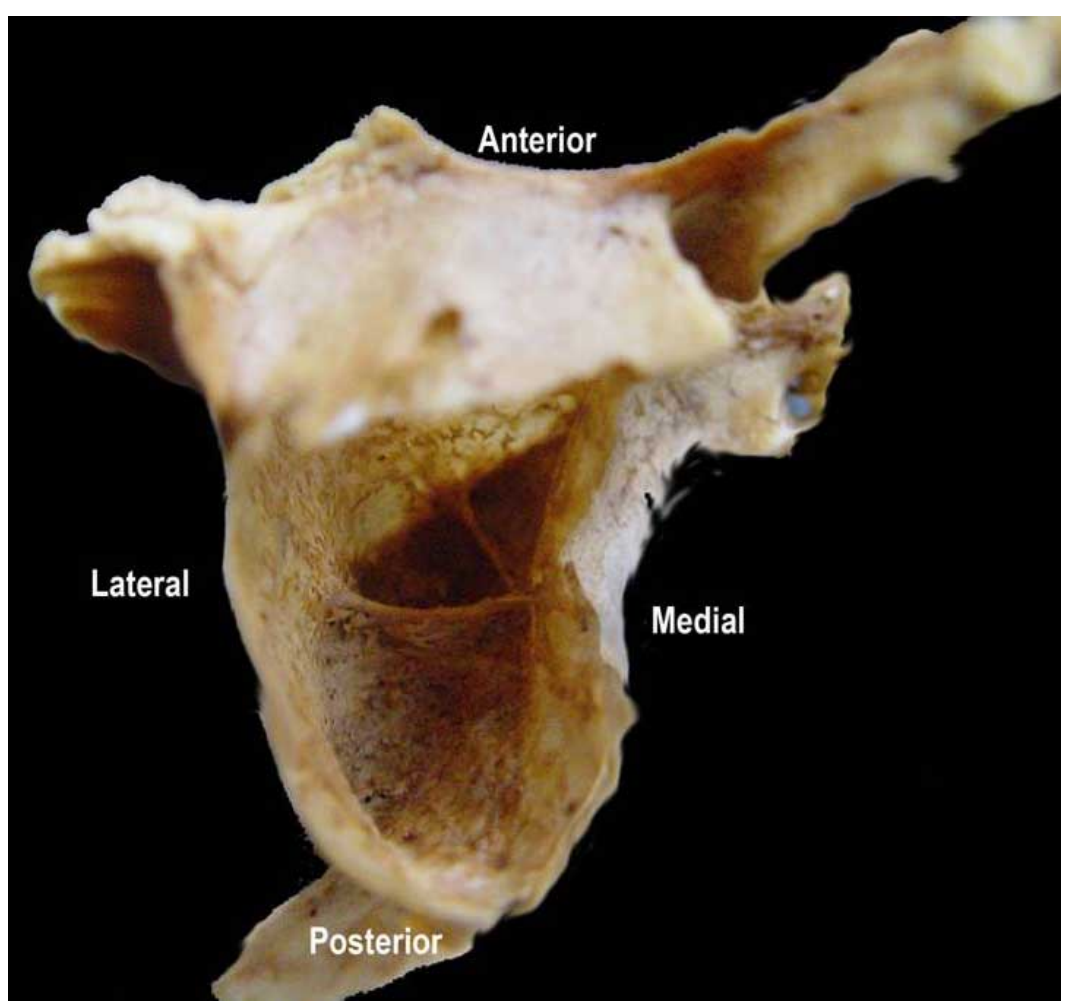

Fig. 1. Hueso maxilar izquierdo aislado con remoción parcial de la porción orbitaria, visto desde superior. Se observa el piso del seno maxilar con la presencia de septos intrasinusales dispuestos desde la pared medial a la lateral.

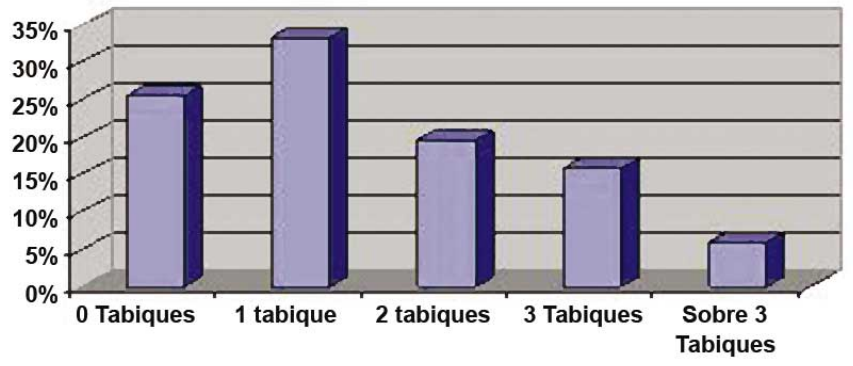

Huesos maxilares según número de tabiques intrasinusales

Fig. 2. Porcentaje de huesos maxilares según cantidad de tabiques intrasinusales.
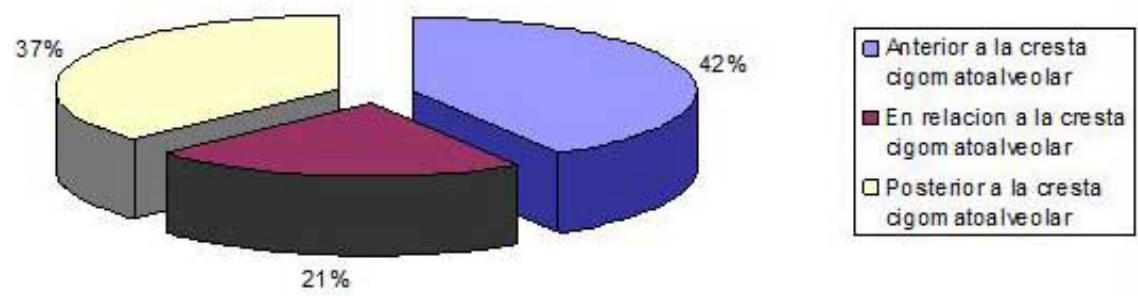

Fig. 3. Porcentaje de distribución de tabiques intrasinusales según división topográfica del piso del seno maxilar. piso del seno maxilar de acuerdo a su división topográfica, demostró que estos se ubican en todas las zonas del piso del seno maxilar en el siguiente porcentaje: En la zona anterior a la cresta cigomato alveolar $42 \%$; en la región de la cresta cigomato alveolar $21 \%$ y en la zona ubicada por posterior a la proyección de la cresta cigomato alveolar 37\% (Fig. 3).

En cuanto a la condición de edentulismo, se estableció que del total de maxilares en condición dentada (42) el $88,1 \%$ de ellos presentó a lo menos 1 tabique; en tanto que del total de maxilares edentulos (9) solo el $11,1 \%$ de ellos presentó un tabique a nivel del piso sinusal.

\section{DISCUSIÓN}

En el presente estudio se evalúo la presencia y distribución de los tabiques intrasinusales en el piso del seno maxilar de piezas óseas de maxilares aislados. Variados autores han demostrado la presencia de estos tabiques (Ella et al.; Gosau et al.; Krennmair et al.; Lee et al.; Shibli et al.; Underwoodal) como también su distribución (Gosau et al.; Kim et al.; Krennmair et al.; MaestreFerrín et al.; Park et al., 2011; Ulm et al.; Underwood).

Los tabiques intrasinusales fueron descritos por primera vez por Underwood, quien al igual que otros autores (González-Santana et al., 2007; Kim et al.; Krennmair et al.; Velásquez-Plata et al.) estudiaron la prevalencia y distribución de los tabiques en el piso del seno maxilar dividiéndolo topográficamente en 3 porciones o áreas: porción anterior definida como la zona premolar, porción media definida como la zona del primer y segundo molar y la zona posterior definida como la 
zona del tercer molar. A diferencia de estos, nuestro estudio propuso utilizar una distribución diferente basándonos en que la distribución en base a ubicación dentaria es engañosa y en muchas ocasiones inexistente, sobre todo si se han evaluado maxilares edentulos cuyas referencias dentarias se han perdido totalmente, esta clasificación es imprecisa e inexacta. De acuerdo a ese razonamiento es que dividimos el piso del seno maxilar utilizando una referencia ósea de fácil distinción como lo es la cresta cigomato alveolar, la que a la vez se asocia a la zona interradicular del primer molar superior.

La prevalencia de los tabiques en el piso del seno maxilar en nuestro trabajo arrojo que estos se encontraban en el 76\% de los huesos estudiados. Tal porcentaje es claramente superior a la encontrada por otros autores (González-Santana et al.; Kim et al.; Krennmair et al.; Park et al., 2011; Shibli et al.; Ulm et al.; Underwood; Velásquez-Plata et al.) quienes obtuvieron en sus respectivos estudios valores entre el 13 y el $58 \%$. Tales diferencias pueden explicarse por el hecho que este trabajo fue realizado sobre piezas óseas secas, sin presencia de mucosa que tapizara el interior del seno maxilar y realizado con una visión directa y ocupando un dispositivo de alta luminiscencia que permitía observar cada uno de los espacios del seno maxilar, mientras que otros estudios fueron realizados en senos maxilares tapizados con mucosa, por lo que aquellos tabiques de menor altura, grosor o extensión pudieran no haber sido considerados, del mismo modo aquellos estudios en que la observación se realizo en piezas óseas al igual que nuestro trabajo, esta fue realizada sin ayuda de un dispositivo de luminiscencia; recordando que el seno maxilar es una cavidad neumática de compleja observación directa, debido a que solo se abre en una pequeña porción de su cara medial (lugar por donde drena), lo cual explicaría el menor porcentaje de tabiques observados. En tanto que aquellos estudios realizados con imagenología utilizando radiografías panorámicas (Gonzalez-Santana et al.; Krennmair et al.; Shibli et al.), y debido a la sobreposición de estos tabiques en la imagen, claramente mostrarían una subestimación en el diagnostico de presencia de tabiques intrasinusales; esta situación claramente no ocurre cuando la observación es realizada mediante el uso de tomografía computarizada (González-Santana et al.; Kim et al; Krennmair et al.; Park et al., 2011; Velásquez-Plata et al.) en donde la presencia de estos tabiques es mayor en comparación a la observación realizada mediante radiografía panorámica (GonzálezSantana et al.; Krennmair et al.).

La realización de cortes a nivel del maxilar por parte de la tomografía computacional, debiera permitir observar la mayor parte de los tabiques a nivel del piso sinusal.
Los cortes realizados por el tomógrafo computacional se dirigen perpendicularmente al eje del proceso alveolar y teniendo en cuenta que los tabiques generalmente se distribuyen desde la pared lateral del seno hacia su pared medial, se puede establecer que en aquellos cortes realizados en el sector posterior del maxilar, muchos de los tabiques pueden ser cortados en su mismo eje por lo cual no serán apreciados como tales, si no como parte del piso sinusal; esta situación no ocurre en el sector anterior en donde aquellos tabiques que se dirigen desde lateral a mesial son cortados transversalmente en relación a su eje mayor y por ende debieran ser observados en las imágenes. Esto explicaría porque existe una diferencia en el porcentaje de tabiques observados en este trabajo en comparación a la de otros autores que estudiaron la prevalencia de los mismos pero mediante tomografía computacional (González-Santana et al.; Krennmair et al.). Sin embargo, este es el examen imagenologico que nos da una visión mas exacta en cuanto a presencia y distribución de los tabiques intrasinusales, sobre todo si el análisis se realiza en forma acuciosa y en los tres sentidos del espacio.

En cuanto al porcentaje de maxilares de acuerdo al numero de tabiques, se estableció que estos presentan principalmente un tabique único alcanzando el $33 \%$ del total de la muestra, estos resultados coinciden con otros estudios (Gosau et al.; Lee et al.) en donde la presencia de un tabique predomina por sobre las otras. Sin embargo en nuestro trabajo encontramos un alto porcentaje de maxilares con 2 tabiques $(19,6 \%), 3$ tabiques $(15,7 \%)$ e inclusive sobre 3 tabiques $(5,9 \%)$, esto difiere con otros estudios (Gosau et al.; Lee et al.) en los que no observaron mas allá de tres tabiques. La explicación se remite a lo descrito en el párrafo anterior en donde se explicaría la subvaloración en la observación de los tabiques por parte de variados autores.

La distribución espacial de los tabiques observados en este estudio, estableció que el $42 \%$ de estos se ubican por anterior a la cresta cigomato alveolar, siendo esta área en donde se ubican principalmente. La distribución de los tabiques en el sector relacionado a la cresta cigomato alveolar fue de un $21 \%$, en tanto que el sector posterior mostró una distribución del $37 \%$ Esto coincide con otros estudios (Krennmair et al.; Ulm et al.) cuya ubicación preferencial fue en el sector anterior. A diferencia de lo encontrado en nuestro trabajo, Underwood observó que la ubicación principal de los tabiques corresponde al sector posterior. Es importante destacar y recordar que los parámetros que fueron ocupados para dividir topográficamente el piso del seno maxilar fueron diferentes a los ocupados en otros estudios, pese a esto la comparación entre los estudios es factible de realizar. 
La evaluación de la presencia de los tabiques intrasinusales en relación a la condición de edentulismo estableció que del total de maxilares dentados (42) en un gran porcentaje $(88,1 \%)$ existió a lo menos un tabique a nivel del piso sinusal, a diferencia de esto, del total de maxilares edentulos (9) solo el $11,1 \%$ presentó a lo menos un tabique a nivel de su piso. Estos valores pueden ser hasta cierto grado discutibles debido a la gran diferencia en cuanto al número de maxilares evaluados dependiendo de la condición de dentulismo y edentulismo (42 maxilares dentados vs 9 maxilares edentulos). En variados estudios (Kim et al.; Krennmair et al.; Lee et al.) se ha establecido que la cantidad de tabiques intrasinusales es mayor en aquellos maxilares cuya condición es la de edentulismo, esto se explicaría por que los tabiques intrasinusales se formarían debido a la neumatizacion del seno maxilar una vez que las piezas dentarias se han perdido. Este tipo de tabique es conocido con el nombre de tabique secundario y esta íntimamente relacionado con la pérdida de dientes y la posterior neumatización del seno maxilar, lo que explicaría el mayor porcentaje de maxilares edentulos bajo esta condición. Sin embargo es valido preguntarse si un maxilar cuya condición de edentulismo de varios años, posterior a la forma- ción de los tabiques secundarios y debido a la constante neumatizacion del seno maxilar finalmente estos también terminen por perderse, por lo que un maxilar con un edentulismo prolongado en el tiempo quedaría con un seno amplio y libre de irregularidades y de tabiques. Esta posible teoría podría explicar los resultados de nuestro estudio en donde solo el $11,1 \%$ de los maxilares edentulos presento algún tabique en el piso del seno maxilar.

En conclusión, la perdida de dientes establece cambios morfológicos tanto a nivel maxilar como a nivel mandibular. La anatomía normal del seno maxilar se ve afectada tanto por su neumatización como por la presencia de tabiques intrasinusales, situación que puede influir en una posterior rehabilitación protésica mediante implantes. En este estudio realizado sobre maxilares secos y aislados se pudo establecer que los tabiques intrasinusales están presentes en gran parte de los maxilares con una frecuencia y ubicación variable. Esta situación deberá ser considerada y evaluada en conjunto con exámenes imagenológicos para establecer un buen diagnostico y de este modo disminuir riesgos en la realización de técnicas quirúrgicas de elevación del piso del seno maxilar.

HERNANDEZ, C. A.; VISTOSO, M. A; HERNANDEZ, Q. R. \& ROJO, P. J. Prescence and distribution of intrasinusal septa in the maxillary sinus floor. Int. J. Morphol., 29(4):1168-1173, 2011.

SUMMARY: The loss of upper teeth causes alveolar process resorption and maxilary sinus pneumatization. Oral implantology has solved these losses, however, when pneumatization of the maxillary sinus exists, bone availability is diminished, difficulting implantology procedure. To remedy this situation, the surgical technique of lifting the maxillary sinus floor is indicated, which can have complications because of the internal maxillary sinus morphology, specifically the presence of intrasinusal septa. The aim of this study is to verify the presence and distribution of intrasinusal septa, due to its importance in surgical techniques performed in oral implantology. We conducted a descriptive study, based on visual analysis of isolated maxilary bones. Of 65 bones, 51 ( 42 dentate and 9 edentulous)were selected that met certain inclusion criteria. The second part of the study was, to divide the sinus floor topographically into three regions: anterior to the zygomatic alveolar crest, in relation to it, and posterior to the crest. The observation of the septa was performed by a single examiner, assisted by a USB device with 4 high-luminance LEDs. Of the 51 maxillary analyzed obtained: $74.5 \%$ had at least one intrasinusal septum, $25.4 \%$ showed no visible septa. The maxilary bones that had a single septum corresponded to $33.3 \%$ of the sample, $19.6 \%$ of the sample had only two septa, $15.7 \%$ had three septa, while the maxillary bones that had more than three intrasinusal septa corresponded only to $5.9 \%$. Of all septa found ( 75 septa) $42 \%$ was observed in the anterior region, $21 \%$ in the region of the zygomatic crest and $37 \%$ in the posterior region of the maxillary sinus. Of the 42 dentate maxillary bones in $88,1 \%$ present intrasinusal septa, while the total edentulous maxillary bones (9) only $11 \%$ present intrasinusal septa. This study establishes that a significant portion of the maxillary bones have intrasinusal septa that modify the morphology of the sinus floor with a variable frequency and location. This situation must be considered and evaluated by imaging techniques in case of performing surgical elevation of the maxillary sinus floor.

KEY WORDS: Maxillary sinus; Intrasinusal septa; Maxillary sinus floor elevation.

\section{REFERENCIAS BIBLIOGRÁFICAS}

Chanavaz, M. Maxillary sinus: anatomy, physiology, surgery, and bone grafting related to implantology--eleven years of surgical experience (1979-1990). J. Oral Implantol., 16(3):199-209, 1990.
Ella, B.; Noble, R. da C.; Lauverjat, Y.; Sédarat, C.; Zwetyenga, N.; Siberchicot, F. \& Caix, P. Septa within the sinus: effect on elevation of the sinus floor. Br. J. Oral Maxillofac. Surg., 46(6):464-7, 2008. 
Farah, G. \& Nafis, A. F. Morphometric analysis of developing maxillary sinuses in human foetuses. Int. J. Morphol., 24(3):303-308, 2006.

González-Santana, H.; Peñarrocha-Diago, M.; Guarinos-Carbó, J. \& Sorní-Bröker, M. A study of the septa in the maxillary sinuses and the subantral alveolar processes in 30 patients. J. Oral Implantol., 33(6):340-3, 2007.

Gosau, M.; Rink, D.; Driemel, O. \& Draenert, F. G. Maxillary sinus anatomy: a cadaveric study with clinical implications. Anat. Rec., 292(3):352-4, 2009.

Greenstein, G.; Cavallaro, J. \& Tarnow, D. Practical application of anatomy for the dental implant surgeon. J. Periodontol., 79(10):1833-46, 2008.

Kim, M. J.; Jung, U. W.; Kim, C. S.; Kim, K. D.; Choi, S. H.; Kim, C. K. \& Cho, K. S. Maxillary sinus septa: prevalence, height, location, and morphology. A reformatted computed tomography scan analysis. J. Periodontol., 77(5):903-8, 2006.

Krennmair, G.; Ulm, C. W.; Lugmayr, H. \& Solar, P. The incidence, location, and height of maxillary sinus septa in the edentulous and dentate maxilla. J. Oral Maxillofac. Surg., 57(6):667-72, 1999.

Lawson, W.; Patel, Z. M. \& Lin, F. Y, The development and pathologic processes that influence maxillary sinus pneumatization. Anat. Rec., 291(11):1554-63, 2008

Lee, W. J.; Lee, S. J. \& Kim, H. S. Analysis of location and prevalence of maxillary sinus septa. $J$. Periodontal Implant Sci., 40(2):56-60, 2010.

Maestre-Ferrín, L.; Galán-Gil, S.; Rubio-Serrano, M.; Peñarrocha-Diago, M. \& Peñarrocha-Oltra, D. Maxillary sinus septa: A systematic review. Med. Oral Patol. Oral Cir. Bucal, 15(2):e383-6, 2010.

McGowan, D. A.; Baxter, P. W. \& James, J. The maxillary sinus and Its dental implications. Oxford, Butterworth-Heinemann, 1993. pp.1-25.

Park, I. H.; Song, J. S.; Choi, H.; Kim, T. H.; Hoon, S.; Lee, S. H. \& Lee, H. M. Volumetric study in the development of paranasal sinuses by CT imaging in Asian: a pilot study. Int. J. Pediatr. Otorhinolaryngol., 74(12):1347-50, 2010.

Park, Y. B.; Jeon, H. S.; Shim, J. S.; Lee, K. W. \& Moon, H. S. Analysis of the anatomy of the maxillary sinus septum using three-dimensional computed tomography. J. Oral Maxillofac. Surg., 69(4):1070-8, 2011.

Raja, S. V. Management of the posterior maxilla with sinus lift: Review of techniques. J. Oral Maxillofac. Surg., 67(8):17304, 2009.
Shibli, J. A.; Faveri, M.; Ferrari, D. S.; Melo, L.; Garcia, R. V.; d'Avila, S.; Figueiredo L. C. \& Feres, M. Prevalence of maxillary sinus septa In 1024 subjects with edentulous upper jaws: A retrospective study. J. Oral Implantol., 33(5):293-6, 2007.

Ulm, C. W.; Solar, P.; Krennmair, G.; Matejka, M. \& Watzek, G. Incidence and suggested surgical management of septa in sinuslift procedures. Int. J. Oral Maxillofac. Implants, 10(4):462$5,1995$.

Underwood, A. S. An inquiry into the anatomy and pathology of the maxillary sinus. J. Anat. Physiol., 44(4):354-69, 1910.

Van den Bergh, J. P.; Ten Bruggenkate, C. M.; Disch, F. J. \& Tuinzing, D. B. Anatomical aspects of sinus floor elevations. Clin. Oral Implants Res., 11(3):256-65, 2000.

Velásquez-Plata, D.: Hovey, L. R.; Peach, C. C. \& Alder, M. E. Maxillary sinus septa: a 3-dimensional computerized tomographic scan analysis. Int. J. Oral Maxillofac. Implants, 17(6):854-60, 2002.

\section{Dirección para Correspondencia: Dr. Arnoldo Alejandro Hernández Caldera Instituto de Ciencias Biomédicas \\ Facultad de Medicina \\ Universidad de Chile \\ Santiago \\ CHILE}

Email: ahernandezc@med.uchile.cl

Recibido : 22-06-2011

Aceptado: 26-08-2011 